\section{Cahiers d'ethnomusicologie}

Anciennement Cahiers de musiques traditionnelles

$8 \mid 1995$

Terrains

\title{
Terrains et terreau d'un musicien
}

The lands of a musician

\section{Marc Loopuyt}

\section{(2) OpenEdition}

\section{Journals}

Édition électronique

URL : http://journals.openedition.org/ethnomusicologie/1198

ISSN : 2235-7688

Éditeur

ADEM - Ateliers d'ethnomusicologie

Édition imprimée

Date de publication : 31 décembre 1995

Pagination : 137-146

ISBN : 2-8257-0537-3

ISSN : $1662-372 X$

Référence électronique

Marc Loopuyt, «Terrains et terreau d'un musicien », Cahiers d'ethnomusicologie [En ligne], $8 \mid$ 1995, mis en ligne le 04 janvier 2012, consulté le 20 avril 2019. URL : http://journals.openedition.org/ ethnomusicologie/1198

Ce document a été généré automatiquement le 20 avril 2019.

Tous droits réservés 


\title{
Terrains et terreau d'un musicien
}

\author{
The lands of a musician
}

\author{
Marc Loopuyt
}

1 Après trente ans de fréquentation assidue de l'Andalousie, du Maghreb et du ProcheOrient, la notion de terrain, privative sur le plan spatial, m'apparaît d'abord contradictoire avec le monde de la musique traditionnelle dont la générosité potentielle sait transcender toutes les frontières apparentes.

2 Premièrement, qui dit terrain dit limites : il y a donc, dans ce cas, un extérieur et un intérieur et donc une nécessité de passage. C'est certainement la situation de départ la plus fréquente: Monsieur Untel habite à Lyon et, pour lui, la musique des Aït Bouguemmez est à trois heures d'avion, plus une journée de voiture tout-terrain. Autrement dit, entre lui et l'objet de son désir musical, il y a un passeport, un billet d'avion, des contrôles magnétiques et des bureaux de change. Encore faut-il qu'il arrive dans le Haut Atlas après la récolte des noix et que son comportement humain soit adaptable en fonction d'un savoir vivre patriarcal donné. Voilà donc un nombre de limites déjà assez considérable bien que notablement inférieur à celui de l'époque de la Croisière Jaune. L'important est que, face à ces obstacles somme toute fastidieux, il y a le désir de Monsieur Untel ; et comment se fait-il que cette personne se trouve dans cet état d'aspiration irrépressible? C'est qu'une effluve de séduction spécifique a mobilisé puissamment son esprit : aujourd'hui, c'est une photo, un enregistrement sonore ou un film; hier, c'était un récit, un objet ou un indigène exceptionnellement de passage. Face à des obstacles naturels chiffrables en kilomètres, altitude et jours de démarches administratives, se mobilise un élément subtil, non mesurable, mais néanmoins fulgurant : une volonté au service d'un besoin esthétique.

Pour en venir à mon modeste cas, mes parents possédaient un disque de Pepe de Almeria, guitariste flamenco de terroir, inconnu en Espagne en dehors de cette localité, mais ayant enregistré à Paris où il s'était installé. L'écoute de ce disque a déterminé mon aspiration pour la guitare. Cette aspiration m'a conduit chez une professeur de guitare classique qui, certes, aimait le flamenco, mais ne le pratiquait que par l'intermédiaire de partitions et 
ne pouvait donc souligner pour moi l'importance de la transmission orale et directe. Tout cela se passait à Antibes et il a fallu un déménagement de toute notre famille à Strasbourg pour que se présente l'étape suivante. Ma détermination a subi une précipitation alchimique : cette fois le «flamenquiste » n'était plus le produit secondaire de l'industrie phonographique, mais une personne en chair et en os: un modeste plâtrier andalou immigré qui, tout simplement, comme il l'aurait fait chez lui, jouait de la guitare pour le plaisir, après le travail, dans un café de son quartier. Son répertoire n'était pas strictement flamenco, puisqu'il jouait le plus souvent des paso-dobles, mais il aimait le répertoire jondo et connaissait quelques belles falsetas de malagueñas et de verdiales. Je n'étais pas alors en position d'analyser ainsi la rencontre, mais le point déterminant fut le son de cet artiste : ses doigts, musclés par le travail manuel, attaquaient la corde avec une franchise et une efficacité fulgurantes et ses notes me transperçaient le corps comme pour se ficher dans le mur derrière moi. C'est-à-dire que quelque chose m'avait définitivement atteint et que, sans le savoir, cet homme avait déterminé en moi la grande quête du son.

4 Rétrospectivement, les questions possibles sont: était-il indigène en terrain exogène, était-il exogène en terrain indigène? Ou, sur un plan plus subtil, n'était-ce pas un indigène qui en découvrait un autre en terrain exogène? Quant à une limite de " terrain ", il ne s'agissait plus que d'une obsession qui était devenue mienne : franchir la douane espagnole. Cela fut fait à maintes reprises, en troisième classe de la Renfe, dans une joyeuse ignorance des usages qui m'a fait trop souvent croquer dans le casse-croûte de modestes voyageurs andalous, ne connaissant pas les convenances et la nécessité chevaleresque de refuser certaines invitations à partager bocadillos $y$ bebidas.

5 Je m'initiais en même temps à la pratique de la transmission orale et à l'exercice d'une apparente indépendance. Ce qui provoquait une ébullition certes positive, mais somme toute assez désordonnée. Le plan sur lequel la notion privative de terroir est la plus vraie est certainement celui des pédagogies. D'un côté, le modèle scolaire moderne où, le plus souvent, l'élève est coupé de l'enseignement du quotidien, et de l'autre, le système traditionnel du compagnonnage où la maturité vient bien plus tôt à cause du contact constant et précoce avec un modèle humain en exercice de sa propre maturité, le tout dans l'ambiance de la démonstration tacite et puissante de l'exemple : l'école pouponne tard, l'apprentissage éveille tôt ; dans un cas, l'élève est coupé et « protégé » du terrain, dans l'autre, il y commence tout de suite son instruction.

6 Andrès était un maître plâtrier fier de son savoir, et cette gloire éclatait dans sa musique. Cet homme modeste, et néanmoins solaire comme ambassadeur tacite de l'ineffable, m'a déterminé au voyage musical; il a été le passeur psychologique de mes premiers itinéraires musicaux : successivement dans la province de Grenade, puis à Madrid et à Cadiz, à la recherche du secret des compases (cycles rythmiques), des falsetas (variations guitaristiques) et en quête du duende. Le duende est le génie de l'inspiration flamenca qui finit théoriquement par céder à une aspiration tenace : aspiration, inspiration, le terrain est peut-être le lieu où l'on respire, c'est-à-dire où la respiration musicale est maximale ; plus précisément, le lieu spatial identifié ou tout autre lieu subtil, spatial et temporel, voire humain, où l'on prend conscience de cette nécessité. Aspiration et inspiration ne sont pas aussi systématiquement symétriques en jeu musical qu'en respiration biologique, mais ce grand mouvement pendulaire commence par un manque, un besoin, une nostalgie qui doit provoquer la rupture nécessaire de l'équilibre initial dans lequel il y a une frontière entre le sujet désirant et l'objet à connaître. Les flamencos ${ }^{1}$ accomplis qui 
vivent professionnellement à Madrid éprouvent le besoin régulier de retourner en Andalousie. Ils déclarent que c'est pour retrouver el aire, c'est-à-dire la respiration musicale profonde.

Fig. 1 : Avec l'orchestre de Moulay M'hmid el Ouhari à Meknès.

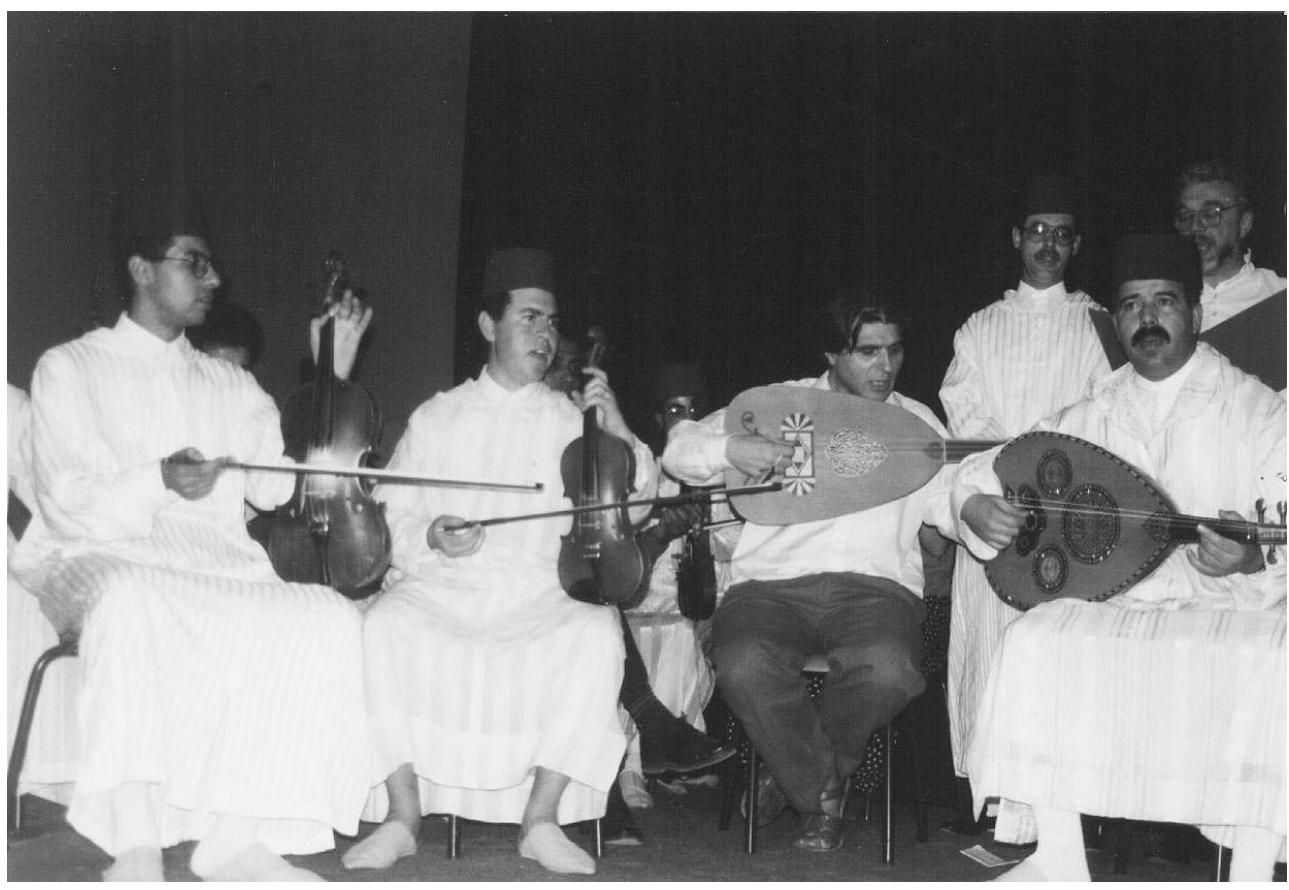

7 Sur quel terrain me sentais-je pendant ces séjours répétés en Espagne ? La continuité, c'était que le décor ressemblait à mon aspiration: minuscule certes vue de l'extérieur, dangereuse même comme pour mes parents alarmés, mais immense vue de l'intérieur. Mon microcosme et ma vision du monde en étaient tout imprégnés. Les séjours alternatifs entre la France et l'Espagne ne sont pas vraiment rupture puisque la guitare est toujours là, cinq, dix et quelques fois douze heures par jour, avec, il faut le dire, plus d'enthousiasme que de méthode. Pour ce qui est de la méthode, l'enthousiasme vocationnel pallie, relativement, au manque d'une tradition de famille ou de clan qui structure dès le plus jeune âge une aspiration artistique. Les chapitres se succèdent et quelques clés se décrochent du grand porte-clé des mystères : révélation sur le système des structures rythmiques : mieux valent trois jours avec Angel Gonzalo Torrès, danseur à Madrid, que trois mois avec tel guitariste du Sud; autrement dit, les nécessaires complémentarités du cuadro flamenco peuvent pallier aux rétentions de savoir dictées par la concurrence et le goût du secret. Ou bien, moi qui à 18 ans ne supportait pas de faire la queue dans les administrations, je me retrouvais après antichambre chez l'alcade ${ }^{2}$ de Cadiz, entrain de garantir que... » Bien sûr, j'ai tel logement à Strasbourg et ce guitariste ici présent, Antonio, y sera hébergé ». (Je croyais que seul moi-même, dans cette aventure, devait changer de terrain). «Eh bien, Monsieur le secrétaire de l'Alcade, c'est Antonio de chez vous qui vient chez moi en France où, tous les deux, nous allons travailler. Que ne ferait-on pas pour une falseta de alegría de Cádiz!» A cette époque, l'Andalousie flamenca me montrait que le terrain ou le terroir conditionne et structure le répertoire, et que répertoire et terroir forgent le récipiendaire humain ; c'est-à-dire que l'art traditionnel, certes, parle de l'artiste mais avant tout, il nous parle du plus intime, du plus profond, du plus poétique, du plus ineffable génie de tel versant, de tel coteau, de 
telle vision grandiose, sous tels oliviers, devant tel hameau perdu, qui rend Paris et les Champs-élysées dérisoires. Un jour, devant Manolo Sanlucar excédé, des étudiants guitaristes du monde entier dissertaient sur l'origine du flamenco : arabe, gitan, séfarade etc. Toutes les thèses y passaient, et la colère du maître gronda : «Quand on laboure un champ andalou, le soc ouvre la terre et le flamenco en jaillit !» Ce fut le terrain, que disje, la terre de Barrameda qui parla par la bouche d'un artiste irréfutablement flamenco: une sentence du maitre de musique, péremptoire, inspirée, efficace et noble qui laissa toute l'assistance coite.

8 J'habitais $30 \mathrm{~m}^{2}$ de soupente à Strasbourg et le « terrain ", c'est-à-dire Antonio, s'y installa avec sa guitare. Pendant quatre mois, les murs résonnèrent de quelques millions de notes, dont quelques centaines passaient d'une guitare à l'autre. Cela dit, l'ambiance psychologique de 1968 et une certaine tendance à la tyrannie du maestrino n'étaient pas compatibles et, avant que les murs ne craquassent, nous avions ré-émigré à Paris où l'obsession du señorito à posséder très vite une Citroën DS 19 fit que nous nous séparâmes. J'avais abrité un pan de l'Andalousie flamenca brute et goûté intra-muros à un mode de vie avec lequel on ne négocie pas. Les prestations professionnelles que nous avions assumées et le goût de la jeunesse pour la vie nocturne avaient rendu l'alliance possible quelques mois, mais le terrain ethnomusicologique transportait avec lui une part de terrain humain incompatible avec le mien.

Soulagement et déception, goût du Sud, ambiance de la « route » et découverte fortuite d'une adresse à Meknès me firent postuler comme enseignant au Maroc. A réception du télégramme d'affectation, j'allai acheter une carte du Maroc et une série de disques de 'oud. Je fis écouter Farid-el-Atrache à un bon ami guitariste et fus presque étonné que cette audition ne le persuadât pas illico d'apprendre le luth oriental, ce qui était déjà le cas pour moi.

10 J'arrivai à Béni-Mellal avec une bonne fièvre, une valise de disques et une guitare et je passai mes premières nuits à l'auberge de jeunesse où répétait un sympathique orchestre arabe avec lequel je fis immédiatement connaissance.

11 Le terrain était submergeant à souhait: parfums, sons, allures humaines, paysages vierges. Déjà l'Andalousie des années 1960 pouvait nourrir l'âme de ce genre de fraîcheur ; mais là, le dépaysement ou le re-paysement était total. En l'occurrence, « repaysement" impliquait pour moi une transplantation dans un paysage rural et harmonieux où la référence à la norme naturelle était submergeante et presque parfaitement écologique avant l'existence de ce terme. Voir construire collectivement et sans argent quatre murs de pisé avec la terre qui est devant, voir confectionner à l'aide d'un simple canif une chalémie avec les roseaux qui sont derrière et entendre jouer à l'intérieur une trentaine d'airs joyeux avec les amis et leurs tambourins : j'ai assisté à tout cela, d'abord complètement ébahi pour constater petit à petit le sérieux et la fragilité de ces savoirs oraux en même temps que secrets. Dans l'Atlas, la nature en bien des lieux n'était qu'égratignée et en retour elle semblait illustrer à souhait la notion amérindienne de Terre-Mère. En particulier pour le musicien, elle fournissait une esthétique de cadre de vie apaisante et inspirante. Je ne veux pas dire que de ce terrain était exclue la sempiternelle lutte pour la vie ou la survie.

12 On pourrait dire que pour un musicien traditionnel, le terme de terrain évoque puissamment la réminiscence proche ou lointaine du terroir à l'état initial avec son terreau, tel que l'art de référence a pu y naître et s'y développer. Le terreau est alors un équilibre écologique, psychique et spirituel. Dans tel contexte qualitatif, l'apparition 
onirique d'un répertoire, sa transmission et son enrichissement par une chaîne d'esprits sains et de mémoires vives coulent de source. Les soufis de l'Atlas parlaient du cœur dans sa cage (que nous appelons thoracique). Le inshirah c'est l'élargissement de la poitrine : l'oiseau est à l'aise et il chante. Le qabd, c'est le resserrement de cette cage qui fait que l'oiseau se prostre.

Dans les années 1968-1971, j'ai donc habité à Foum-l-ançeur, village du Moyen Atlas marocain. Je ne pourrais pas m'attarder ici sur les vertus poétiques du paysage avec le torrent, les grandes falaises et les sources ${ }^{3}$, mais plutôt essayer de décrire quelques caractéristiques du paysage de l'âme musicale.

euple de ce terroir est la tribu des Aït Saïd ou Ali, peuple africain du nord, berbère et musulman baigné dans le soufisme, c'est-à-dire porteur de traditions harmonieusement concommittantes. Deux pôles dans l'ambiance y étaient très vite perceptibles : la règle en même temps que la tolérance pour la "marge humaine ${ }^{4}$. Cette étonnante détente de l'état psychologique de l'homme quotidien a été pour moi l'objet d'une curiosité soutenue. Certes, les musiques étaient intensément présentes, mais où est la cause et où est l'effet? Elles-mêmes semblaient bénéficier d'une source d'inspiration faisant référence. Premier indice: les enfants se transmettent les airs de flûte, le soir, assis autour de la source et leur musique est éminemment "liquide». Autre indication explicite celle-là : le maître Abdeslam Ougoujil me déclare : «Prends ton guembri (petit luth), pars seul et marche le long de l'eau... » Indication explicite certes, mais qu'un rat qui n'est ni tout à fait des champs, ni tout à fait des villes, médite toujours après des années : avoir l'oreille assez vierge pour que l'eau puisse nous enseigner des airs ou nous transmettre un point capital de l'art musical... De plus, l'indication pourrait bien concerner non seulement les torrents d'Aït Saïd ou Ali, mais encore ceux de tout terrain où coulent des ruisseaux. Tristes ruisseaux et tristes terrains que ceux qui ne trouvent aucun souffleur de chalumeau à qui dicter les airs!

15 A ces indices plus que précieux succède la découverte d'une clé, je veux dire le phénomène qui explique le mieux cet extraordinaire sentiment d'hospitalité, de fraternité, de grâce et de tolérance qui caractérise l'âme et la musique des Aït Saïd : la danse extatique et son instauration mythique chez les soufis Kadiri d'Aït Saïd ou Ali. Voici la tradition locale qui relate la genèse du hautbois, ghaïtah, et qui explique le fait que cette danse mystique (la hadrah) est paradoxalement appelée «la danse du diable ». Iblis, le diable, fatigué de son extériorité, voulut un jour pénétrer au Paradis. Il essaya d'en ouvrir la porte qui résista, et il ne put que l'entrouvrir. En s'entrouvrant, elle grinça et un wali (un saint) perçut ce grincement. A la suite de cette révélation sonore, il indiqua comment construire la ghaitah. Ainsi le timbre de l'instrument n'est pas flatteur, il évoque plutôt de prime abord un déchirement violent de nature douloureuse. Quand l'oreille s'y accoutume, les aspects positifs du son se révèlent. C'est un son qui peut emporter toutes les préoccupations mesquines; c'est un son qui peut libérer chacun du durcissement de l'individualité : c'est le son de la catharsis. Dans le village, chaque jeudi, on va au bain pour se purifier le corps et, à l'occasion des fêtes, toute la tribu en cortège marche, joue du bendir (tambour sur cadre) et chante derrière la ghaitah et les tambours, comme pour se purifier l'âme. Alors, ce n'est plus un certain nombre d'hommes, de femmes et d'enfants qui marchent ensemble, mais une seule tribu, une seule famille, une entité ressentie et connue comme unique dans la certitude du cœur. Ainsi Aït Saïd ou Ali, dans un hâl (état mystique) puissant et collectif, semble-t-il réintégrer la dimension indiquée par le nom d'Ali, celle de la connaissance ésotérique. Il s'agit là d'une liquéfaction 
alchimique des egos : chacun ne se connait plus qu'en tant qu'infime partie d'un tout, puis comme réceptacle vide dont les dernières parcelles d'individualité ont été balayées et dissoutes pour un moment par la plénitude et la fulgurance du son de la ghaïtah devenue foudre, tornade et révélation de l'unité cosmique: fraternité humaine (une formule de politesse courante est «mon frère » plutôt que « Monsieur») et proximité, pour ne pas dire parenté, avec la terre nourricière. C'est évidemment un grand thème commun aux traditions chamaniques comme celles des Amérindiens ou des Yakoutes de Sibérie 5 . Pour qui y prête attentivement l'oreille, le son de la ghaitah est celui de la foudre, du déchirement d'un tissu. Dans le cadre du soufisme, le sama' est la production et l'audition musicales ayant pour but de faire se déchirer les « 70000 voiles » qui séparent l'homme de la vérité ; ou pour un musicien, les cloisons qui séparent l'oreille du son primordial, ou de ce que Pythagore appelait la musique des sphères. Cela ne signifie pas que seule la ghaïtah aurait cette fonction et cette seule fonction. Dans un autre cadre et selon d'autres mondes esthétiques, il s'agira du nay, du setâr, du tanbur etc.

Fig. 2 : Avec ses élèves chez Mounir Bachir à Baghdad.

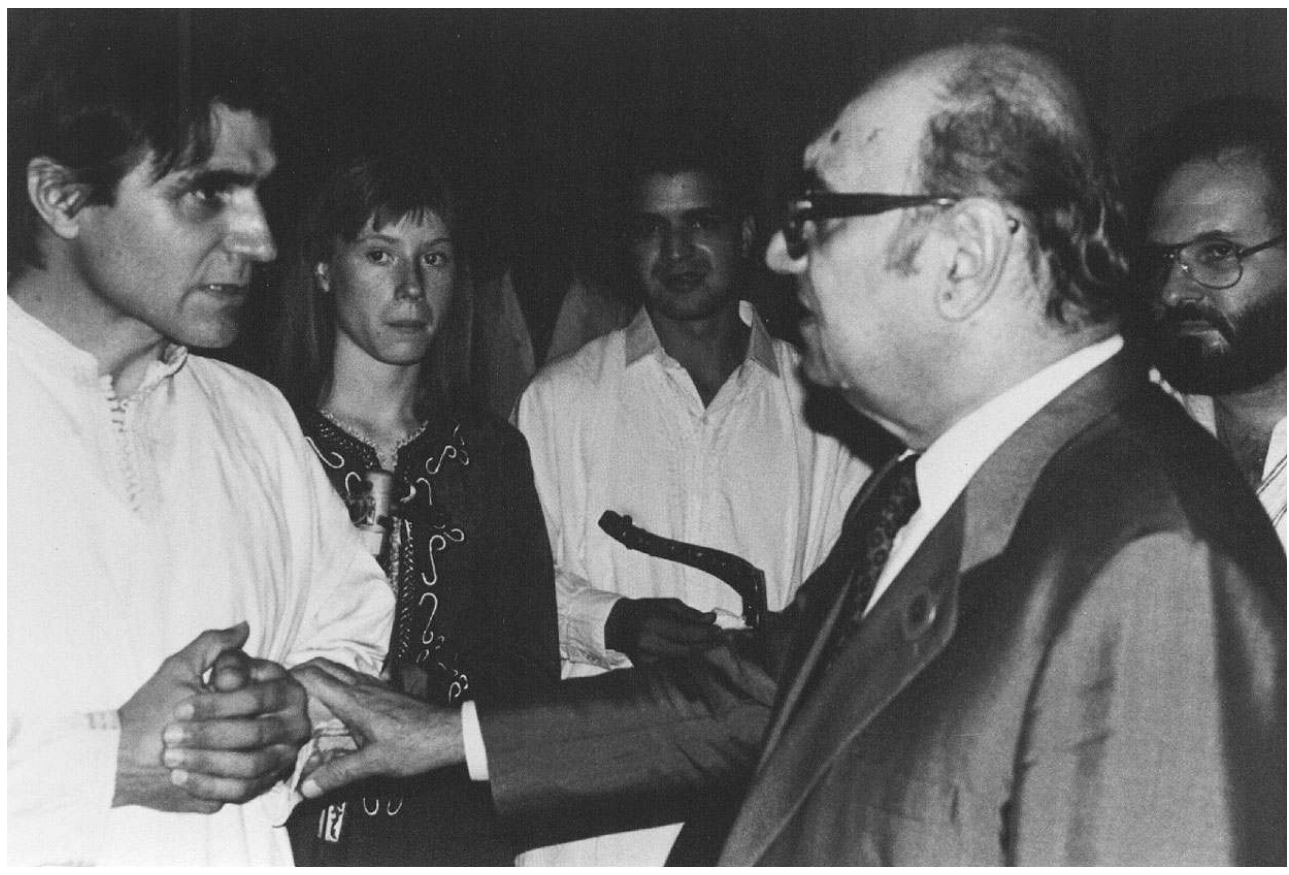

Après le cadre champêtre et montagnard de l'Atlas, je m'installai pour quatre ans à Fès où je pus continuer à fréquenter le milieu des musiciens traditionnels toujours proches du soufisme et de la préoccupation subtile et spéculative de l'art ${ }^{6}$. Dans ce cadre essentiellement citadin, la référence à la nature normative est d'une part le patio avec sa fontaine et ses plantations et d'autre part le symbole de l'organisation modale qui est l'Arbre des Modes. Ces deux points illustrent la continuité de principe entre ces deux «terrains », mais les modes de fonctionnement s'avèrent bien différents. Si l'Atlas est une montagne ouverte, Fès est une ville à remparts et si, quelque part, j'ai eu la sensation des frontières d'un terrain musical, c'est bien là. Rétrospectivement, j'analyse mieux la situation : j'y venais pour apprendre la tradition du 'oud et je frappais à la porte de la plus vénérable institution de musique citadine arabo-andalouse, axée sur le chant, sans recommandations, avec une nationalité française et un accent marocain de terroir. Analogiquement, ce fut donc comme si un provincial à passeport marocain frappait à la 
porte du Conservatoire de Paris; d'où une certaine méfiance initiale de l'établissement dont j'ai été finalement élève pendant trois ans. Cette vigilance jalouse est à la mesure du sens de la responsabilité dans la transmission. Ce qui se transmet c'est bien sûr un corpus immense de poèmes et de musique, mais c'est aussi l'intuition de la destination subtile des nouba-s. Ustad Massano Tazi ${ }^{7}$, héritier de Mtahiri, suggérait efficacement cette préoccupation sous-jacente à son art qui s'affirmait dans la zaouyah ${ }^{8}$ où sa musique menait la hadrah ${ }^{9}$ vigoureuse qui, bousculant tous les cloisonnements apparents, ramenait dans l'Atlas, ou tout simplement au centre de soi-même ou encore à la racine de l'Arbre des Modes. Il est certain qu'à Fès, un musicien peut trouver une explicitation ou en tout cas quelques clés efficaces pour l'approfondissement du sens de sa disposition naturelle, pour peu qu'il soit sensible à la suggestion et à l'implicite.

Je passai avec ma famille une dernière année marocaine à Chechaouen dans le cadre humainement austère des Jbala. Un soufi de Zemmij, près de Tanger, à qui je confiais mon inquiétude à l'idée de devoir quitter le terrain marocain pour le terrain français me répondit dans un bon rire éloquent: pour lui, il n'y avait qu'un terrain, le terrain intérieur de l'âme, et cette translation spatiale n'était qu'accident. Je rentrai donc en France avec une bonne fièvre, peut-être la même que celle de mon arrivée en 1968, et, une fois opérés les rétablissements d'usage après une longue absence, continuai mes tribulations en musique traditionnelle.

D'abord, mouvement pendulaire oblige, je fis venir en Europe à plusieurs reprises les musiciens d'Aït Saïd ou Ali et Ustad Massano Tazi, ainsi que ceux des Aït Bouguemmez du Haut Atlas, vallée-témoin de l'antique civilisation berbère que je découvris à cette occasion avec le maitre bouganim ${ }^{10} \mathrm{Ahmed} \mathrm{Aïssa}^{11}$ : horizons lointains et toujours la même certitude, celle de revenir chez moi.

19 Après la vigueur du 'oud marocain, j'aspirai aux raffinements du 'oud turc, et une pièce de Cinuçen Tanrikorur écoutée sur une cassette d'amateur me mobilisa une année sur sa piste: je trouvai sa trace, aux détours de mes concerts à Bangkok et le rencontrai finalement à Bruxelles. Ce magnifique «pan de terrain » traditionnel prit l'habitude de séjourner chez moi à Nancy à l'occasion de ses tournées et voyages. J'organisai l'enregistrement de son disque à Paris ${ }^{12}$ et séjournai bien des fois chez lui, dans la ville d'Ankara dont l'apparence extérieure n'évoque en rien un terrain en harmonie esthétique avec la prodigieuse érudition du maître dans l'art du maqam turc.

Après avoir passé un certificat d'aptitude ouvert à la musique traditionnelle, je fus invité à enseigner le 'oud à l'Ecole Nationale de Musique de Villeurbanne en France, ce qui, aujourd'hui, me permet de découvrir une imbrication étonnante de terrains culturels existant sur l'agglomération. Par le biais des élèves issus de différentes communautés maghrébines ou orientales, je découvre une vie musicale traditionnelle qui sait organiser les tournées de ses artistes de renom sur le terrain public ou le terrain privé. C'est ainsi que j'ai pu rencontrer tranquillement Samy el Maghrebi sur place, moi qui n'avais pu espérer l'entrevoir au Maroc, ou constater comment d'excellents et authentiques musiciens algériens musulmans sont détenteurs d'airs emblématiques exclusivement réservés à des familles de Juifs originaires d'Algérie. C'est aussi la rencontre avec des musiciens arméniens et le phénomène de l'évolution de leur langage musical depuis la diaspora de 1915. Grâce à eux, Yeghish Manoukian, maître du hautbois doudouk est en France et nous enregistrons à la maison un répertoire délaissé en Arménie depuis quarante ans : le mugham. Pour une autre partie du travail, j'emmène le groupe près d'un ruisseau dans une forêt de l'Isère et le dialogue s'instaure aussitôt avec l'eau et les 
oiseaux, comme dans l'Atlas, comme dans le Caucase mythique, comme dans les miniatures ou comme dans l'imagination poétique ${ }^{13}$.

Voilà évidemment pourquoi j'ai indiqué plus haut que je ne voyais plus guère les limites des terrains musicaux stricto-sensu. Le terrain c'est d'abord un regard ou un angle sous lequel on perçoit le monde musical et qui fait que les barrières géographiques, historiques ou ethniques s'estompent. Les terrains mythiques semblent globalement en voie de disparition, mais les artistes qui souffrent le plus du naufrage esthétique que cela implique, s'efforcent d'en ressaisir les arcanes musicales, indépendamment de leur origine: Ali Akbar Khan lui-même a dit que bientôt certains aspects du raga indien n'existeraient plus qu'en Occident.

Le terrain est le jardin où pousse l'Arbre des Modes ${ }^{14}$. Cet arbre a quatre branches qui correspondent aux quatre humeurs de l'homme et aux quatre qualités du cosmos. Dans ses racines se cache le mode "gharibat el muharrar", mot à mot, étranger (au monde ordinaire, donc extraordinaire) et libérateur. Il est libre de la condition des humeurs et, comme les racines, il fonctionne sur un plan caché.

O lecteur, prête bien attention car voici la fin du conte: le jour, cet arbre est dressé comme tous les arbres, mais la nuit, sa cime pointe vers le sol et ses racines vers le ciel, car tout musicien qui joue et tout public qui écoute ne se rencontrent que pour sortir de l'état de la grisaille ordinaire commune à tous les terrains.

BIBLIOGRAPHIE

\section{Références bibliographiques}

LOOPUYT Marc, 1983, Aspects musicaux du Moyen-Atlas. Etudes traditionnelles 84/481 : 119-126. LOOPUYT Marc, 1988, L'enseignement de la musique arabo-andalouse à Fès. Cahiers de musiques traditionnelles $1:$ « De la bouche à oreille » :39-45.

\section{Références discographiques}

(Les noms cités sont ceux des auteurs des livrets)

DURING Jean, 1994, Ouzbékistan. Monâjât Yulchieva. Maqam d'Asie centrale 1. Ouzbékistan, Ferghâna. Paris : CD Ocora C 560060.

LECOMTE Henri, s.d. (1994), a Sibérie 2. Yakoutie : épopées et improvisations. Sakha. Paris : CD Buda Records 92565-2.

LECOMTE Henri, s.d. (1994), b Sibérie 3. Kolyma : chants de nature et d'animaux. Cukc, Even, Jukaghir. Paris : CD Buda Records 92566-2.

LOOPUYT Marc, 1988, Maroc. Ustad Massano Tazi. Musique classique andalouse de Fès. Paris : CD Ocora C 559035. 
LOOPUYT Marc, 1989, Maroc. Moyen-Atlas : musique sacrée et profane. Paris : CD Ocora C 559057.

LOOPUYT Marc, 1994, Turquie. Cinuçen Tanrikorur, ud. Paris : CD Ocora C 580045.

LOOPUYT Marc, s.d. (1992), Maroc. Musiques de la haute montagne. Aït Bou Guemmez. Paris : CD Buda Records 92580-2.

LOOPUYT Marc, s.d. (1994), Arménie. Sources. Yeghish Manoukian. Gagik Mouradian, Araïg Bartikian. Paris : CD Buda Records 92580-2.

\section{NOTES}

1. Flamencos signifie « flamenquistes».

2. Alcade signifie maire.

3. Voir disque Moyen Atlas: musique sacrée et profane (Loopuyt 1989) ainsi que l'évocation fournie dans l'article « Aspects musicaux du Moyen-Atlas » (Loopuyt 1983)

4. Expression de Frithjof Schuon

5. Voir les disques Lecomte s.d (1984) a et b.

6. Voir Loopuyt 1988.

7. Voir disque Loopuyt 1988.

8. Maison de confrérie soufie.

9. Danse extatique.

10. Boughanim signifie joueur de clarinette double en roseau.

11. Voir disque Loopuyt s.d. (1992).

12. Voir disque Loopuyt 1994 (1986).

13. Voir disque Loopuyt s.d. (1994).

14. Voir livret du disque Loopuyt 1988.

\section{RÉSUMÉS}

Plutôt qu'un article, ce texte est la relation d'un intinéraire dont les étapes sont des terrains voisins de musiques traditionnelles de la Méditerranée. Marc Loopuyt nous décrit sa rencontre avec le flamenco en plusieurs phases : les disques, un immigré andalou, l'Andalousie elle-même et, enfin, un guitariste flamenco de terroir qui immigre avec lui en France. C'est ensuite sa découverte du Maroc qui commence par l'Atlas avec ses vertus de proximité de la nature-norme et Fès qui intègre spéculativement ce type de savoir à son architecture des nouba araboandalouses. Le périple se poursuit en Turquie et le mouvement se stabilise apparemment à Villeurbanne où les activités de l'auteur lui font explorer les jardins secrets des musiques traditionnelles de la Méditerranée en France. La soif d'intimité avec les terrains explorés fait que la perspective sur le terrain d'origine se transforme et que l'auteur y pose à plusieurs reprises un regard délibérément « huron ».

This is not so much an article but a text linked to an itinerary, the stages of which are the neighbouring traditional musical areas of the Mediterranean. Marc Loopuyt describes his encounter with flamenco through several steps : records, an Andalusian immigrant, Andalusia 
itself and finally a flamenco guitarist of the Andalusian heartland who emigrates with him to France. Following that, it's his discovery of Morocco, beginning in the Atlas mountains which enjoy the virtue of nature-norm proximity and on to Fez which encompasses in a speculative way this type of knowledge with its nouba Arab-andalusian architecture. The journey is furthered in Turkey and things stabilise, it would seem, in Villeurbanne, where the author explores the musical traditions of Mediterranean in France. The desire for intimate knowledge of the lands explored, leads the author to adopt a quite deliberate layman's approach on several occasions.

\section{AUTEUR}

\section{MARC LOOPUYT}

Marc Loopuyt est né dans les Deux-Sèvres en 1947. A 15 ans, il débute l'étude de la guitare flamenca avec un immigré espagnol. Il séjourne régulièrement en Andalousie. A 20 ans, il s'installe au Maroc où il étudie le 'oud. Il se perfectionne ensuite en Turquie et au Proche-Orient arabe. Il est aujourd'hui enseignant et responsable du département des musiques méditerranéennes au Conservatoire de Musique de Villeurbanne. Outre de nombreuses émissions sur les radios européennes et des articles sur les traditions musicales, il a assuré la direction artistique, réalisé les livrets de cinq disques de musique traditionnelle et signé trois disques de son propre travail instrumental, tous remarqués par la critique : $4 \mathrm{f}$ à Télérama, Diapason d'Or, 5 Diapasons et trois fois Choc du Monde de la Musique. 